\title{
New, affordable, open-hardware neutron monitor electronics
}

\author{
Krüger, PP; Krüger, HG; Krüger H.; Diedericks, C; Malan, D. \\ Center for Space Research, North-West University \\ E-mail: Helena.Krugerenwu.ac.za
}

\begin{abstract}
The Center for Space Research has been operating four neutron monitor (NM) stations since the 1960s and has also developed mini neutron monitors, initially to calibrate the stations. To upgrade the aging electronics in the NMs, a new electronic system has been developed.

The aim of the new electronic design is to be modular so that it can be easily adapted to stationary or mobile NMs. The system uses commercial-off-the-shelf components and publicly-available software where possible. The software and hardware designs will be made freely available to the international NM community. The system is also designed for easy maintenance and calibration. It is affordable, shielded against electromagnetic interference and it makes the data available on the internet in real-time.

The electronic system also features a fast acquisition system for pulse shape recording and microsecond accurate event timestamps. The pulse profiles are used for calibration and integrity testing to ensure long term stability. The accurate timestamps make multiplicity and time-ofarrival studies possible.
\end{abstract}

Some first measurements with the new electronic system are presented and compared to previous systems. 


\section{Introduction}

A worldwide network of neutron monitors (NMs) is used to record variations in cosmic rays. The NMs only count the number of cosmic-ray events above a certain threshold rigidity and can not discriminate between cosmic rays of different rigidities. To get a cosmic-ray spectrum, NMs at different latitudes are used. The Center for Space Research (CSR) has been operating four NM stations at different latitudes, in Antarctica, Hermanus, Potchefstroom and Tumeb, for several decades as part of the international network of neutron monitors.

Because the count rate of each NM station depends on the station's configuration and environment, a calibration neutron monitor has been developed to inter-calibrate the stations [5]. Due to their relatively low cost, these monitors are now used as semi-autonomous neutron monitors, called MiniNMs [6]. However, it has been found that the count rate of the MiniNMs is quite sensitive to temperature and high voltage variations due to small variations in the threshold level. The previous MiniNM electronic heads are not very easy to upgrade or adapt to other neutron monitors.

It has long been known ([3]) that a single neutron monitor station can give some information about the cosmic-ray spectrum. Higher energy CRs on average result in more neutrons generated in the NM (called multiplicity). Modern electronics, however, now makes it possible to make use of this information, by measuring the time delay between consecutive events $([1,2])$. Using a single neutron monitor, the energy spectrum of a GLE ([4]) and the spectrum hardening during a Forbush decrease ([8]) has been measured. The dependence of the time delay distribution on the cosmic-ray spectrum was demonstrated by a latitude survey [2] and by Monte-Carlo simulations [7].

Due to the aging electronics of the four CSR neutron monitor stations, the old electronic systems will be gradually upgraded. A new, modular head was developed that can be used on both the MiniNMs and large stationary NMs. The new head records the pulse profile and exact arrival time of each event generated by the NM as described in Section 1. The capabilities of the new head are demonstrated in Section 2, showing that it can not only do multiplicity analysis, but that the pulse profile information can be used to improve the calibration of the neutron monitor.

\section{Electronic head}

The aim of the development of the new head was to

- be able to record the pulse profile and exact arrival time with sub-microsecond accuracy.

- use affordable, commercial off-the-shelf components as far as possible.

- keep the design modular so that it can be used on different NMs and so that it is easy to adapt and upgrade.

- make the design freely available to the broader scientific community by using freely available design software, open-hardware and open-source software.

Figure 1 shows the main components of the electronic head:

1. Like a conventional NM head, it has to supply high voltage to the detector tube.

2. When a neutron is captured by the tube, the electric field in the tube results in an electron avalanche, giving an small output pulse. This pulse needs to be amplified and recorded. 


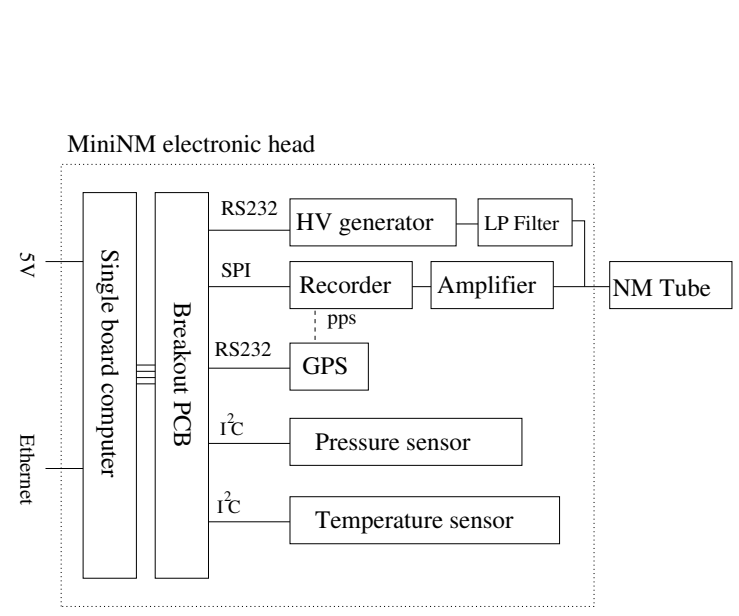

(a) System Diagram

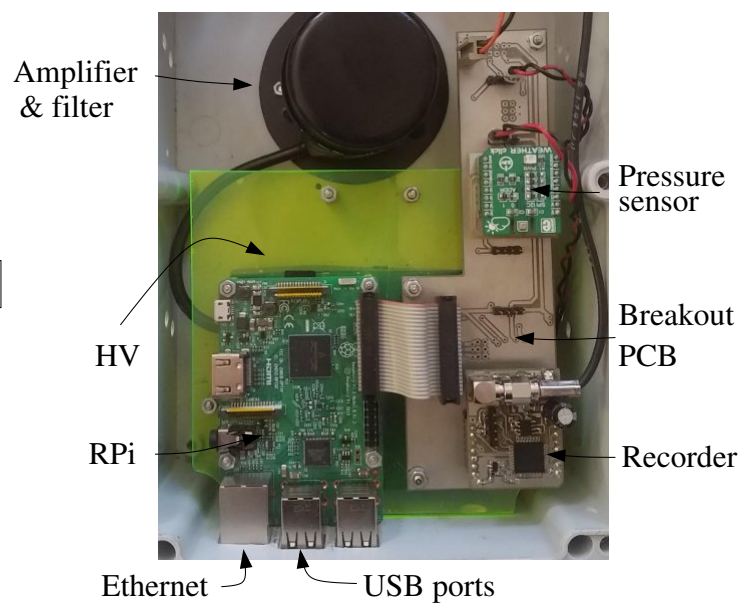

(b) Photo

Figure 1: The MiniNM electronic head

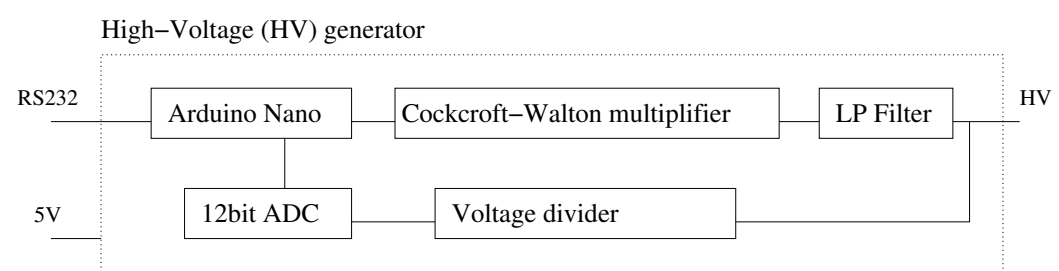

Figure 2: System diagram of high-voltage generator

3. To be able to perform pressure and temperature corrections, the MiniNM also needs an accurate pressure sensor and temperature sensor.

4. Some MiniNMs also need to record the position of the monitor using a GPS module.

5. The MiniNMs must also be able to upload their data to the NMDB in real-time if an internet connection is present.

\subsection{Single-board computer}

The well-known Raspberry Pi (RPi) single-board computer (SBC), running the open-source operating system Raspbian is used as the main computing unit and the other components are developed as plug-ins where each plug-in has its own driver running on the Raspberry Pi. When a new component is added or an existing component is upgraded, only the corresponding driver has to be installed or updated. A breakout PCB is used to connect the various components to the RPi and to distribute power to the various components. When the SBC is upgraded, only the breakout PCB has to be adapted.

\subsection{HV generator}

Buying commercial high-voltage generators was found to be either very expensive or the high voltage was not accurately enough controlled. A simple HV generator was developed (see Figure 
2) that uses an Arduino Nano that controls a Cockcroft-Walton multiplier to generate the required voltage. An accurate $1 \mathrm{G} \Omega$ resistor, 12-bit ADC and oversampling are used to measure the output voltage to an accuracy better than $0.5 \mathrm{~V}$. The high voltage can be controlled from the SBC by giving the HV generator a command of the required voltage. The HV generator also periodically returns the actual voltage to the Raspberry $\mathrm{Pi}$, which is logged.

\subsection{Amplifier}

A pulse amplifier (using a single BF998 transistor) and an operational amplifier (MCP662) were used to amplify the pulse generator by the tube. The amplifier also acts as a bandpass filter, to filter out low-frequency noise $(<34 \mathrm{kHz})$ generated by the capacitors as well as high-frequency noise ( $>1 \mathrm{MHz}$ ) generated by the amplifier. It was found that any high-voltage capacitors used in the head generated low-frequency noise when there was mechanical vibration due to the capacitor's electromechanical properties.

\subsection{Recorder}

A simple and affordable digitiser and pulse recorder was developed by using an PIC $32 \mathrm{MZ}$ microprocessor. Although these microprocessors have 12-bit ADC modules that can together reach a sample rate up to $18 \mathrm{MHz}$, it currently only samples 10 bit at $2 \mathrm{MHz}$. All the samples above a certain threshold are recorded and sent to the Raspberry Pi together with a timestamp through the microprocessor's fast SPI or UART port. When a GPS PPS signal is supplied to the microprocessor, the timestamp is linked to absolute GSM time.

\subsection{Additional sensor}

There is a wide variety of commercial sensors that can be connected to the Raspberry Pi. The breakout PCB has space for three additional sensors and four USB ports are available on the Raspberry Pi.

For the MiniNM, affordable Bosch pressure sensors (BME280) are added that measure pressure, temperature and relative humidity. The accuracy of these sensors is still being investigated, but preliminary results show that the pressure accuracy is better than $0.1 \%$.

\section{Results}

Two MiniNMs, one with a ${ }^{3} \mathrm{He}$ tube and one with a ${ }^{10} \mathrm{BF}_{3}$ tube (see [6]) were fitted with the new electronic heads.

Using the pulse profiles of each event, the amplitude (height) and width of each pulse were calculated. A normal amplitude (pulse height) histogram (Section 3.1) and a two dimensional amplitude-width histogram (Section 3.2) are useful tools to calibrate the head and to monitor the performance of the neutron monitor. The arrival time of each event can also be used in multiplicity studies, such as constructing a time delay distribution (Section 3.3). 


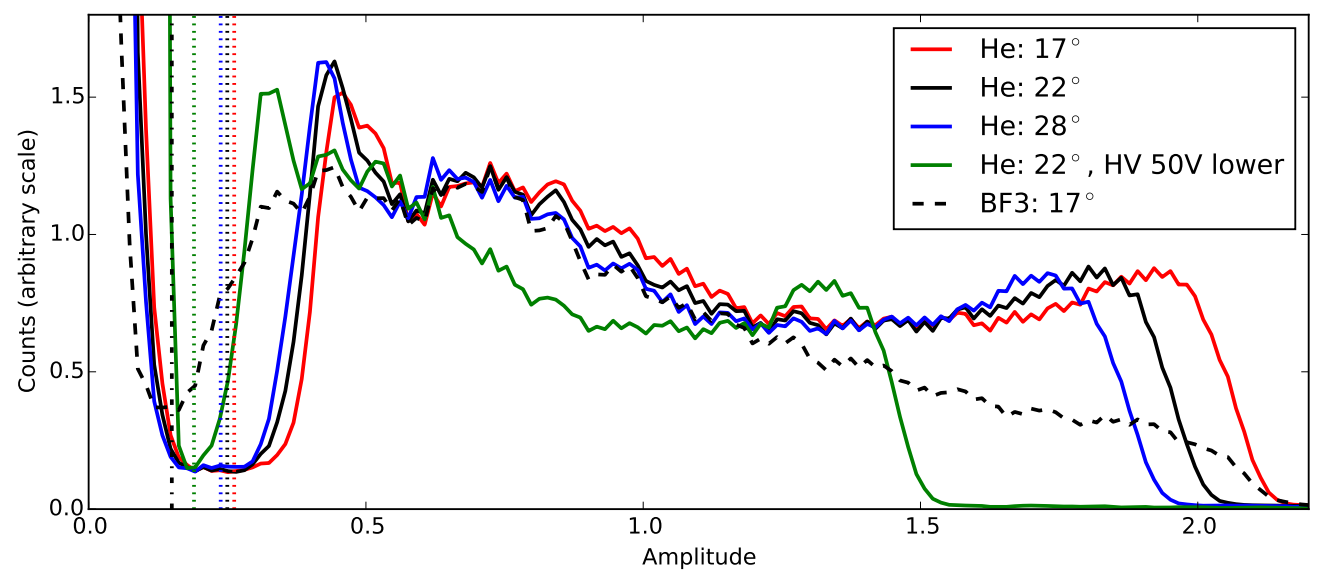

Figure 3: Pulse amplitude histogram of the MiniNMs using 24 hours of data.

\subsection{Pulse height histogram}

Fig 3 shows the pulse amplitude histogram for the ${ }^{3} \mathrm{He}$ and ${ }^{10} \mathrm{BF}_{3}$ MiniNMs using one day of data. Using these diagrams, a threshold level (dotted lines) is determined to discriminate between noise (below the threshold) and CR events (above the threshold). In the old NM heads, this threshold level was kept fixed and only events above the threshold were counted. By recording the amplitude of each pulse and by applying the threshold cut afterwards (during post-processing) it is now possible to adapt the threshold level as conditions, such as gain or noise, change. For example, when the temperature changes by $5^{\circ} \mathrm{C}$, the gain of the amplifier changes by $5 \%$ as shown in Figure 3. If the threshold is kept fixed as in the old MiniNM head, the change in gain will result in a $0.1 \%$ change in the count rate of the $\mathrm{NM}$, i.e. a temperature coefficient of $-0.02 \% /{ }^{\circ} \mathrm{C}$. However, when the threshold is adapted, the count rate becomes gain independent.

The histograms are also useful to detect problems in NMs, such as a drift in high voltage. The high voltage is kept constant using a feedback loop. However, should there be something wrong with the feedback, Figure 3 shows how a small change in high voltage (3\% lower) would look like a large change in gain (35\% lower), which can easily be detected. If the threshold is not adapted, it will lower the count rate by about $1 \%$.

\subsection{Pulse amplitude-width histogram}

One graph that is powerful in monitoring the performance of a NM is a 2D histogram of the width and height of all the pulses. Figure 4 shows two such graphs for a MiniNM with a ${ }^{3} \mathrm{He}$ tube. The arc on the left is the electronic noise and the triangular shape at the bottom is the characteristic curve of the tube. The discrimination between noise and events can be improved by not only using a cut in amplitude $(>0.25 \mathrm{~V}$ ) but also a cut in width ( $<45$ samples) as shown by the dashed lines. This results in a $0.4 \%$ lower count rate, due to less noise being counted. It also reduces the sensitivity of the count rate to variations in the amplitude threshold level. For example, a $20 \%$ variation in the amplitude threshold will result in about a $0.5 \%$ variation in the count rate when no width threshold is used, but a $0.4 \%$ variation in the count rate when the threshold is included. 


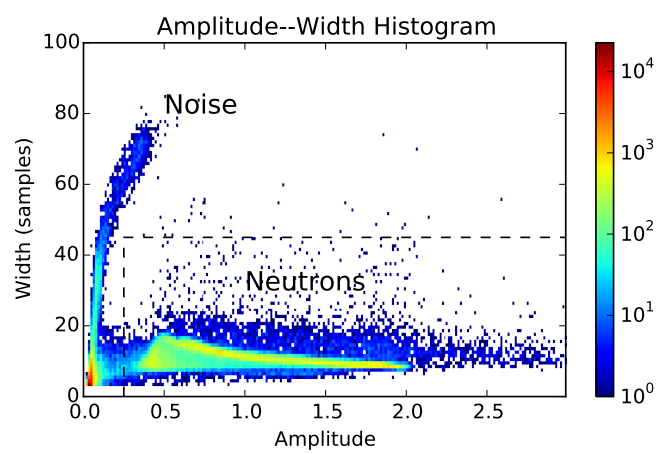

(a) MiniNM

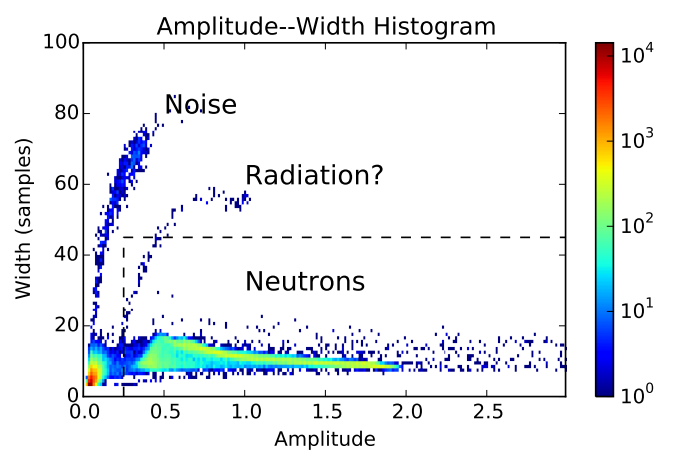

(b) Bare tube

Figure 4: Pulse amplitude-width histogram of a MiniNM with a ${ }^{3} \mathrm{He}$ tube. The dashed lines show the discrimination levels.

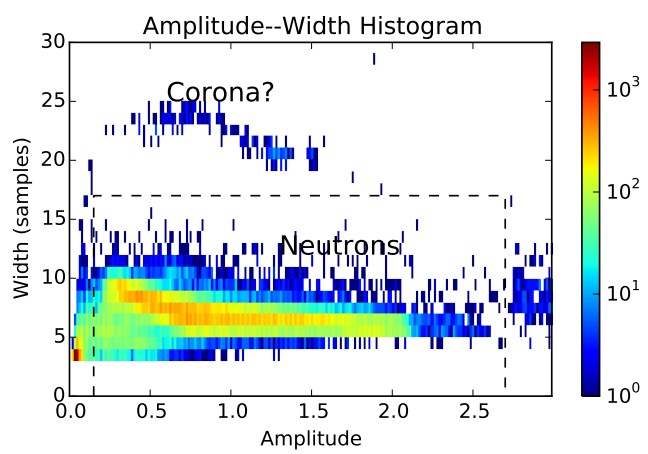

Figure 5: Pulse amplitude-width histogram of a faulty MiniNM with a ${ }^{10} \mathrm{BF}_{3}$ tube. The dashed lines show the discrimination levels.

The amplitude-width histogram is also very useful to detect and analyse problems with the neutron monitor. For example, Figure $4 \mathrm{~b}$ shows the histogram of a bare ${ }^{3} \mathrm{He}$ tube without lead or moderator. Another type of event is visible, which is probably some background radiation that is normally shielded by the lead. Figure 5 shows the $2 \mathrm{D}$ histogram for a faulty ${ }^{10} \mathrm{BF}_{3} \mathrm{NM}$. The top arc and events on the right is noise, probably due to some corona on the HV line. In this case, the bad events can be cut out using the dashed rectangle. This problem would not even be noticeable is events are only counted with a conventional NM head.

\subsection{Time Delay Distribution}

A histogram of the time delay between consecutive events can be used to analyse the multiplicity of CR events $([1,2])$. The new electronic head records the exact time of each event, making it possible to calculate such a histogram. [1,2] shows that the time delay distribution (TDD) has a Poisson component and an excess below $1 \mathrm{~ms}$ which can be fitted by using the sum of two exponential functions (Figure 6). They propose that the two time constants are determined by the lifetime of evaporating neutrons $(\mathrm{t}=370 \mu \mathrm{s})$ and the average neutron detection time. 


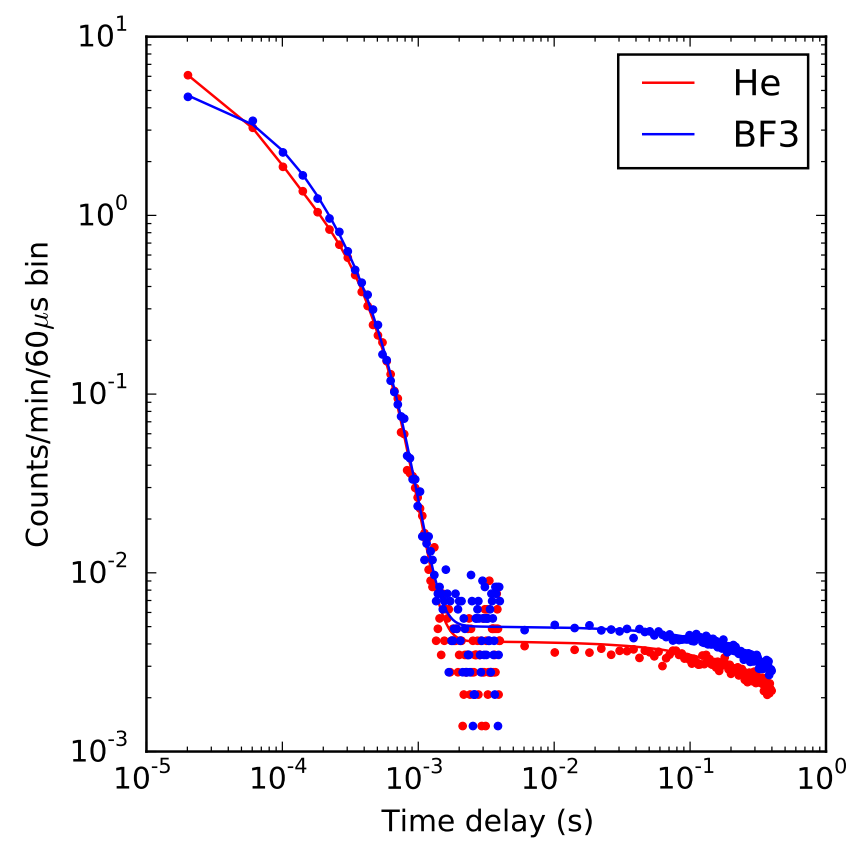

$w(t)=N_{0} e^{-t N_{0}}+\frac{a_{1}}{\tau_{1}} e^{-t / \tau_{1}}+\frac{a_{2}}{\tau_{2}} e^{-t / \tau_{2}}$

\begin{tabular}{|c|c|c|c|}
\hline & ${ }^{3} \mathrm{He}$ & ${ }^{10} \mathrm{BF}_{3}$ & \\
\hline \hline$N_{0}$ & 92.6 & 104.6 & Counts/minute \\
\hline$a_{1}$ & 5.5 & 4.7 & \\
\hline$\tau_{1}$ & 36 & 69 & $\mu \mathrm{s}$ \\
\hline$a_{2}$ & 11.1 & 10.7 & \\
\hline$\tau_{2}$ & 208 & 209 & $\mu \mathrm{s}$ \\
\hline
\end{tabular}

Figure 6: Measured and fitted time delay distribution of MiniNMs.

Fig 6 shows the TDD of the ${ }^{3} \mathrm{He}$ and ${ }^{10} \mathrm{BF}_{3}$ MiniNM using only one day's data, fitted using a Poisson distribution and two exponential functions. Note that two different bin sizes where used for time delays smaller and larger than 4 ms. However, this does not influence the goodness of the fit, as a maximum log-likelihood fit was done. The first time constant is found to be about $210 \mu \mathrm{s}$ for both monitors and the second time constant is about $37 \mu$ s for the ${ }^{3} \mathrm{He} \mathrm{NM}$ and $70 \mu \mathrm{s}$ for the ${ }^{10} \mathrm{BF}_{3} \mathrm{NM}$. The reason for the difference is that the diameter of the ${ }^{10} \mathrm{BF}_{3}$ tube is almost double that of the ${ }^{3} \mathrm{He}$ tube. The same lead rings are used, so that the ${ }^{10} \mathrm{BF}_{3}$ tube has less moderator material between the tube and the lead, resulting in the neutrons being moderated more by the reflector. The reflector is on the outside of the lead, which is farther away, resulting in a larger detection time. However, the fraction of multiplicity events (see $a_{1}$ and $a_{2}$ ) is similar between the two monitors, but slightly larger in the ${ }^{3} \mathrm{He}$ monitor, probably due to more moderator material.

\section{Conclusion}

Using modern electronics, an affordable NM electronic head was built to record the pulse profile for each event. It is easy to upgrade and is adaptable to different neutron monitors.

The pulse profile information can be used to improve the calibration of the NM head and better monitor the performance of the NM:

- The pulse amplitude information can be used to adapt the discrimination level for drifts in gain and high voltage in order to minimise the effect these drifts have on the count rate. This reduces the temperature dependence of the count rate due to the electronics. 
- It was shown that the pulse width can be used to better discriminate between noise and CR events. Using the pulse shape should make it possible to further improve the discrimination. This needs further investigation.

- We found that an 2D histogram of pulse width and pulse height is a powerful diagram to investigate the performance of a NM, as different types of events have different signatures.

The new head also make it possible to do multiplicity analysis, which makes the NM capable of determining the CR spectrum to some extent.

The design of the electronic head makes use of open-hardware and open-source software in order to make it accessible to the broader scientific community.

\section{References}

[1] Y.V. Balabin, B.B. Gvozdevsk, E. A. Maurchev, E. V. Vashenyuk, and D. D. Dzhappuev. Fine structure of neutron multiplicity on neutron monitors. Astrophys. Space Sci. Trans., 7:283-286, 2011.

[2] J. W. Bieber, J. M. Clem, M. L. Duldig, P. A. Evenson, J. E. Humble, and R. Pyle. Latitude survey observations of neutron monitor multiplicity. Journal of Geophysical Research, 109:A12106, 2004.

[3] E. Dyring and B. Sporre. Latitude effect of the neutron multiplicity as detected by a shipborne neutron monitor. Arkiv foer Geofysik (Sweden), 5, 1966.

[4] B.B. Gvozdevsky, Y.V. Balabin, E.V. Vashenyuk, and L.I. Schur. Neutron multiplicity measurements in barentsburg during december 13, 2006 GLE. In Physics of Auroral Phenomena, Proc. XXXI Annual Seminar, Apatity, pages 119-121, 2008.

[5] H. Krüger, H. Moraal, J. W. Bieber, J. M. Clem, P. A. Evenson, K. R. Pyle, M. L. Duldig, and J. E. Humble. A calibration neutron monitor: Energy response andinstrumental temperature sensitivity. Journal of Geophysical Research, 113:A08101, 2008.

[6] H. Krüger, H. Moraal, R. Nel, H.G. Krüger, and M. O'Kennedy. The mini neutron monitor programme. In 34th ICRC, 2015.

[7] P.-S. Mangeard, D. Ruffolo, A. Sáiz, W. Nuntiyakul, J. W. Bieber, J. Clem, P. Evenson, R. Pyle, M. L. Duldig, and J. E. Humble. Dependence of the neutron monitor count rate and time delay distribution on the rigidity spectrum of primary cosmic rays. Journal of Geophysical Research: Space Physics, 121(12):11,620-11,636, 2016.

[8] D. Ruffolo, A. Sáiz, P.-S. Mangeard, N. Kamyan, P. Muangha, T. Nutaro, S. Sumran, C. Chaiwattana, N. Gasiprong, C. Channok, C. Wuttiya, M. Rujiwarodom, P. Tooprakai, B. Asavapibhop, J. W. Bieber, J. Clem, P. Evenson, and K. Munakata. Monitoring short-term cosmic-ray spectral variations using neutron monitor time-delay measurements. The Astrophysical Journal, 817(1), 2016. 